\title{
INVESTIGATION ON THE AHA-EXPERIENCE AS AN INDICATOR OF CORRECT SOLUTIONS IN FUNCTIONAL ANALYSIS IN ENGINEERING DESIGN
}

\author{
Zimmerer, Christoph; \\ Nelius, Thomas; \\ Matthiesen, Sven \\ Karlsruhe Institute of Technology
}

\begin{abstract}
The functional analysis of technical systems is an important part of the design process. To further improve the design process, especially the functional analysis, it must not be viewed as a monodisciplinary process. To this end, cognitive factors such as the aha-experience must also be included in studies of analysis processes to a greater extent. This paper investigates the relationship between the occurrence of aha-experiences and the correctness of solutions in the analysis of a technical system. An aha-experience is a strong feeling of subjective certainty that accompanies the cognitive process of suddenly finding a previously unknown solution. For this purpose, a study on the functional analysis was evaluated. The results show that many identified subfunctions of the system under investigation were identified with an aha-experience and that these subfunctions are more often correct. The results also suggest that aha-experiences occur more often among students than among experienced design engineers. Especially among students, a positive relation of aha-experiences on the correctness of the identified subfunction can be seen. This offers potential for further investigations to make aha-experiences useful in design methods.
\end{abstract}

Keywords: Design cognition, Human behaviour in design, Design engineering, functional analysis, aha-experience

Contact:

Zimmerer, Christoph

Karlsruhe Institute of Technology (KIT)

IPEK Institute of Product Engineering

Germany

christoph.zimmerer@kit.edu 


\section{INTRODUCTION}

The functional analysis of technical systems is a central part of the design process. Both in the development of new technical products and in problem-solving, profound knowledge of initially unknown relationships between embodiment, behavior and function is necessary. This knowledge is gained during phases of functional analysis. Wrong or insufficient functional analysis often leads to costly iterations in the development process (Meboldt et al., 2013). Previous concepts to improve functional analysis often focus on identifying successful approaches (Matthiesen and Nelius, 2018a). However, the functional analysis can also be understood as a cognitive process. When analyzing technical systems, designers take up information, interpret it and store it in mental models. Still, there is no comprehensive understanding of cognitive processes in design (Cross).

Thus, to further improve the design process, especially the functional analysis of technical systems, it must not be viewed as an isolated and monodisciplinary process (Gericke and Blessing, 2012). Therefore, the cognitive factors of cognitive processes must also be examined more closely. To this end, design research is increasingly incorporating factors from the field of psychology into studies of design processes (Chrysikou and Gero, 2020). One such cognitive processes that influences the gain in knowledge is the aha-experience. The aha-experience, also known as the eureka effect, is a euphoric experience in which a sudden insight is gained into an initially unclear context (Bühler, 1907). The impact of the aha-experience is that a realization accompanied by an aha-experience is more likely to be correct (Danek and Salvi, 2018). The aim of this paper, therefore, is to investigate the effect of ahaexperiences on the correctness of solutions gained during the functional analysis.

\subsection{Functional analysis in engineering design}

During the design process, a technical system is conceived and all information required for its realization is defined (Bock, 1955). The unique aspect of designing is the anticipation of structures that are supposed to realize a function. The designer changes between qualitatively different solution spaces (Hacker, 2002). The function to be fulfilled is transformed into an embodiment by a synthesis. In a further analysis step, it is verified whether the chosen embodiment fulfils the intended function. The synthesis is based on the designer's idea of how the function can be fulfilled. The knowledge of these interrelationships is especially important in problem-solving but is often only partially known. Without this knowledge, the embodiment is therefore created in iterative sequences of analysis and synthesis (Matthiesen, 2011).

There are different types of analysis. A frequently used type is functional analysis, which describes the identification of the functionality of a technical system. It is used in the further development of existing products, the analysis of competitive products and the training of new employees (Booth $e t$ $a l ., 2015)$. Functional analysis has already been frequently examined with traditional investigation methods such as protocol and document analysis (Booth et al., 2015; Eckert et al., 2012; Matthiesen and Nelius, 2018a) as well as with eye tracking (Hess et al., 2018; Matthiesen and Nelius, 2018a; Ruckpaul et al., 2015). In these studies, the participants are confronted with technical systems whose exact structure and detailed functionality is unknown to them. The goal of the functional analysis is to gain an understanding of how the system works.

Engineering designers gain their knowledge of unknown relationships between embodiment and function during analysis phases. The analysis is therefore of great importance for the design and the entire product development. The importance of analysis can best be described by the effects of an insufficient analysis. An insufficient analysis leads to wrong assumptions. If estimates and assumptions made are falsified at a later stage, this results in iterations (Meboldt et al., 2013; Wynn and Eckert, 2017). While small iterations represent important learning cycles, larger, so-called crossgate iterations can have a significant impact on project resources (Meboldt et al., 2013). Studies of development projects have shown that most bad decisions can be traced back to insufficient analysis (Badke-Schaub and Frankenberger, 1999). However, this implies that a thorough analysis is essential for a satisfying design result (Nelius et al., 2020).

\subsection{The aha-experience}

When analyzing technical systems, designers take up information, interpret it and store it in mental models. The functional analysis can therefore be understood as a cognitive process. In psychology, a sudden realization is called insight, which is often accompanied by an aha-experience. The aha- 
experience is described by Bühler (1907) as a euphoric experience in which a sudden insight is gained into an initially unclear context. Furthermore, insights that are accompanied by an aha-experience occur with a strong feeling of subjective certainty for the solution found (Danek et al., 2014). A wellknown example of an "aha-experience", also known as the eureka-effect, is Archimedes' discovery of the Archimedean principle with the exclamation "eureka", which gave this effect its name.

In psychology, the effect of the aha-experience has been studied in more detail and it has been found that until an aha-experience occurs, different phases occur:

A prerequisite for the occurrence of an aha-experience is that gathered information does not fit the current mental model of a problem. This leads to a cognitive conflict, which results in a mental impasse for the problem solver (Danek et al., 2013). In this mental impasse, the conflict between the received information and the mental model cannot be overcome. During the mental impasse, the problem-solving process comes to a nearly complete halt or the same activity is repeated several times (Jones, 2003). In order to overcome this impasse, the mental model must be restructured. The sudden overcoming of the mental impasse by the process of restructuring is called insight. Such an insight is often accompanied by an exclamation like "Aha" of the problem solver. (Bilalic et al., 2019)

An important feature of insights that are accompanied by an aha-experience is that they are accompanied by an increased sense of subjective certainty about the solution achieved (Danek et al., 2014).

In addition to the increased subjective certainty, there is also evidence from several studies that solutions accompanied by an aha-experience are more precise from an objective point of view (Danek et al., 2014; Salvi et al., 2016). Danek and Salvi (2018) call this effect the "accuracy effect".

On the other hand, there are also situations in which an insight accompanied by an aha-experience leads to a wrong solution (Danek and Wiley, 2016; Laukkonen et al., 2020). False insights are usually accompanied by a high feeling of subjective security due to the aha-experience. This increases the certainty in a false solution and can lead to false solutions being retained and not questioned. Laukkonen et al. (2020) refer to this phenomenon as the dark side of eureka. Decisive for the correctness of a found solution that was accompanied by aha-experiences is the knowledge that was restructured during the restructuring process. If this underlying knowledge is deficient, the solution found is probably also wrong. (Laukkonen et al., 2020)

\subsection{The aha-experience in design}

One limitation that prevents direct transferability to design is the structure of the investigations from which the findings were derived. The presented investigations on aha-experiences from the field of psychology use so-called insight problems as a task. These are short tasks such as anagrams (Laukkonen et al., 2020) or arithmetical problems (Bilalić et al., 2019) for example, where it is assumed that the solution can only be achieved having an insight. Another difference to problems from the field of design is the possibility to directly check the correctness of solutions for insight problems. The solution of the "Nine-Point-Problem" (see Figure 1), where the points shown must be connected by 4 uninterrupted lines, can be checked by the participant very easily. The difficulty with this task is that participants tend to make additional restrictions. For example, the participants assume that the square (right) must not be exceeded. The findings of an analysis problem in the field of design, in turn, often cannot be verified directly by the participant.

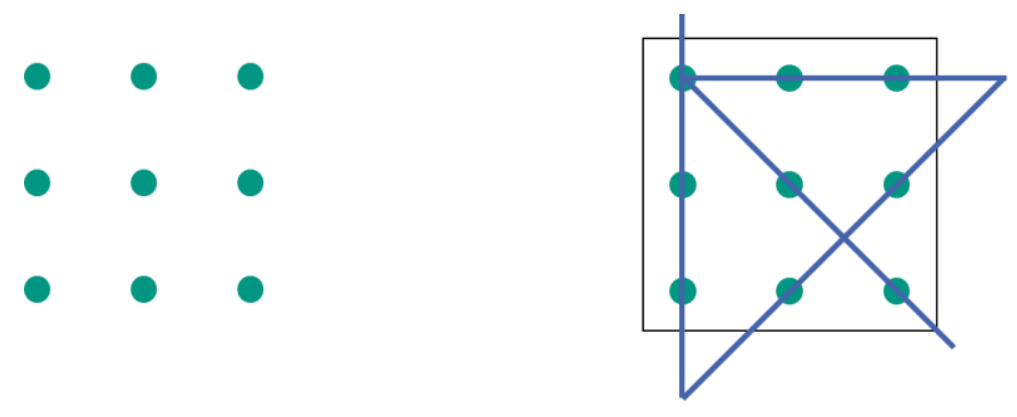

Figure 1. Example of an insight task and its solution (right) (according to Akin and Akin (1996))

The acquisition of aha-experiences is possible by different examination methods. Besides the possibility to let participants self-report on aha-experiences, there is the possibility of identifying aha-experiences 
by independent evaluators using concurrent think aloud protocols (Ericsson and Simon, 1993). With the concurrent think aloud method, the researchers can understand what the participant is thinking about, what he or she is doing, or whether questions arise. This method is established to investigate ahaexperiences (Fleck and Weisberg, 2004; Ash et al., 2012; Cranford and Moss, 2012).

Akin and Akin (1996) have already demonstrated the principle transferability of insight problems that trigger aha-experiences in the field of architectural design. This has been achieved by transferring a classical insight problem from psychology into a task for concept generation. Thereby a well-known insight problem was investigated for the triggers of aha-experiences. These triggers were called Frames of Reference. One trigger identified in the nine-point problem (Figure 1) is the notion that the lines to be drawn must be inside the box. This idea, and thus the trigger, was successfully transferred to a task for concept generation of a building facade. (Akin and Akin, 1996)

Overall, it seems that aha-experiences could also be an important factor in problem solving in product development. However, It has not yet been investigated to what extend aha-experiences occur in the design process and how they relate to the correctness of the solution achieved. Findings from psychological research mostly derive from studies with insight problems and can only be transferred to design research to a limited extent for two reasons. First, insight problems are structured in such a way that the final solution can be achieved in only one step and not in several partial steps as is the case in design problems. Second, in insight problems it is not examined whether finding the solution is really accompanied by an aha-experience, but it is presupposed. Besides that, there is also a risk of a wrong solution, since the classical insight problems do not reflect the reality of the design process enough to transfer the results properly.

\subsection{Objectives}

In psychology, the mental impasse is used as an indicator for an aha-experience. To investigate ahaexperiences in design problems as well, it is necessary to identify a suitable indicator that allows the detection of aha-experiences. The focus will be on functional analysis as an essential part of the engineering design process. One way to identify aha-experiences could be the mental impasse which is used in the field of psychology.

The aim of this paper, therefore, is to investigate the effect of aha-experiences on solutions gained during the functional analysis. Further, it shall be investigated whether aha-experiences are preceded by a mental impasse. For this purpose the following research questions will be addressed:

RQ1: How do situations accompanied by aha-experiences relate to the correctness of solutions in functional analysis?

RQ2: Are there differences regarding the duration to identify a subfunction between situations with and without an aha-experience that indicate a mental impasse?

As differences between novices and experts were found in previous studies (Ruckpaul et al., 2015), both students and experienced design engineers will be considered here as well.

\section{MATERIALS AND METHODS}

\subsection{Participants}

A total of 33 participants took part in the study. These consisted of 18 mechanical engineering students and 15 design engineers with industrial experience.

A requirement for students to participate was that they had completed at least the fourth semester of their studies and had also successfully passed the mechanical design course so that all participants had sufficient basic knowledge to complete the task. The 18 students consisted of 15 male and three female participants. The average age of the students was 23.6 years $(\mathrm{SD}=2.00)$ and the average duration of their studies was 8 semesters $(\mathrm{SD}=2.46)$.

The design engineers came from companies in various industries, for example, the automotive industry, special engineering, engineering services, and drive system technology. The mean work experience of the design engineers was 11.8 years $(\mathrm{SD}=7.77)$ and the mean age was 36 years $(\mathrm{SD}=6.69)$. All participating design engineers were male. 


\subsection{Task}

The functional analysis of a lawn sprinkler was selected as a task. The advantage of this task was that it has already proven to be suitable for the analysis of functions in earlier investigations (Matthiesen et al., 2017; Matthiesen and Nelius, 2018a, 2018b). The aim of the task was to analyse the central gear unit of the lawn sprinkler and to determine how the swivel mechanism works. The swivel mechanism included 10 subfunctions (SF), the most important of which will be briefly presented in the following: The swivel mechanism is driven by a turbine wheel, which generates a rotational movement at high speed using the hydraulic flow (SF1: generate rotation). This rotary motion is then reduced to slow speed by a gearbox (SF2: reduce rotation speed) and braced against the housing (SF3: brace rotation). This causes the entire gear unit to rotate. The swivel range is set by two adjustment rings on the housing of the lawn sprinkler (SF4: limiting swivel range). If the gear unit hits the adjustment rings, a switch is flipped which changes the water flow to the turbine and thus reverses the direction of rotation (SF5: reverse rotation direction).

To complete the task, the participants had 10 minutes of time. In advance, they were given the task description and a short introductory video showing a lawn sprinkler in action. The participants were given two different models of the lawn sprinkler to complete the task. One completely assembled and one additional dismantled gear unit. In addition, the participants had pen and paper for taking notes.

To evaluate the result of the analysis, the think aloud statements of the participants were used. For this purpose, the statements about the individual subfunctions were identified and compared with the actual functioning by the coders.

\subsection{Data collection}

To capture the approach of the participants, they were instructed to use the concurrent think aloud method while working on the task. This method, in which all thoughts should be spoken out aloud, has already been successfully used in earlier studies of aha-experiences (Fleck and Weisberg, 2004). To introduce the participants to the use of the method, they were given a short training video before the actual task started and then a short exercise on how to use it. To ensure that all the participants' statements would be recorded during the processing of the actual task, they were filmed during this phase.

\subsection{Data analysis}

In the first step, the videos with the think aloud statements were transcribed and assigned a timestamp for further analysis. The transcripts were then used to record all statements that could be assigned to one of the relevant subfunctions and thus contributed to the overall understanding of the main function of the lawn sprinkler. Afterward, the identified statements on the subfunctions were classified into the categories correct solution and incorrect solution on the basis of criteria defined in advance of the evaluation.

In a second loop, all statements were examined again. This time the classification was made as to whether the finding of the subfunction was accompanied by an aha-experience or not. As an indication of this, verbal signs of an aha-experience (e.g. aha, ah yes, Ahh!) and the euphoria with which the subfunction was found were used.

In addition, the duration that has elapsed since the previous subfunction was named was determined for each named subfunction using the assigned timestamp.

To answer research question 2, the Mann-Whitney $U$ test is used to find statistical differences in the data. Statistical analysis was performed using IBM's SPSS Statistics 26 and a significance level $p$ of 0.05 was applied.

To increase the quality of the coding, the evaluation was performed by two independent coders and the match was determined using interrater reliability. Cohen's Kappa is used as a measure of interraterreliability (Cohen, 1960). Cohen's Kappa is more robust than a simple match percentage because the probability of a random match is included in the calculation. (McHugh, 2012)

Cohen's Kappa was calculated using the following formula:

$$
K=\frac{P_{0}-P_{c}}{1-P_{c}}
$$

$P_{0}$ is the observed agreement and $P_{c}$ is the expected chance agreement between the two coders. 


\section{RESULTS}

Altogether, 239 statements on the relevant subfunctions were mentioned by the 33 participants during the evaluation of the think aloud protocols. The classification reveals that altogether 28 ahaexperiments could be identified by both coders. Additionally, there were 15 more aha-experiences, which were identified by only one of the two coders. These were not included in the further evaluation. The interrater reliability is $K=0.75$. According to Landis and Koch (1977) this corresponds to a substantial level of agreement.

\subsection{Correctness of solutions}

In 28 out of 239 cases in total an aha-experience could be identified with the subfunction. This means that $12 \%$ of the subfunctions are identified when accompanied by an aha-experience. Overall, it can be said that only 1 out of a total of 28 mentioned subfunctions which were accompanied by an ahaexperience was incorrectly. This leads to an error rate of only $4 \%$ for situations accompanied by an aha-experience. With 37 wrongly identified subfunctions, the error rate of $18 \%$ is considerably higher for the remaining 211 subfunctions.

Figure 2 presents an overview of the mentioned subfunctions clustered according to the occurrence of aha-experiences and the correctness, split into students and design engineers.

What particularly stands out in the figure is that the rate of aha-experiences is twice as high among students $(15 \%)$ as among the more experienced design engineers $(8 \%)$. The number of correctly identified subfunctions with "aha" is also higher among students. There was only one wrongly identified subfunction among the design engineers and among the students even none at all.

When comparing the rate of correct identified subfunctions with and without aha within the group of students (left), it can be clearly seen that subfunctions identified with aha are considerably more often correct than subfunctions identified without aha. While the latter is wrong in $21 \%$ of all cases, the error rate for subfunctions with aha is $0 \%$.

Among the design engineers (right), on the other hand, there seems to be no proportional difference between the categories with aha and without aha in terms of the rate of correctness. This is mainly due to the fact that the subfunctions identified without an aha-experience are correct to a considerably higher degree than is the case among the students.

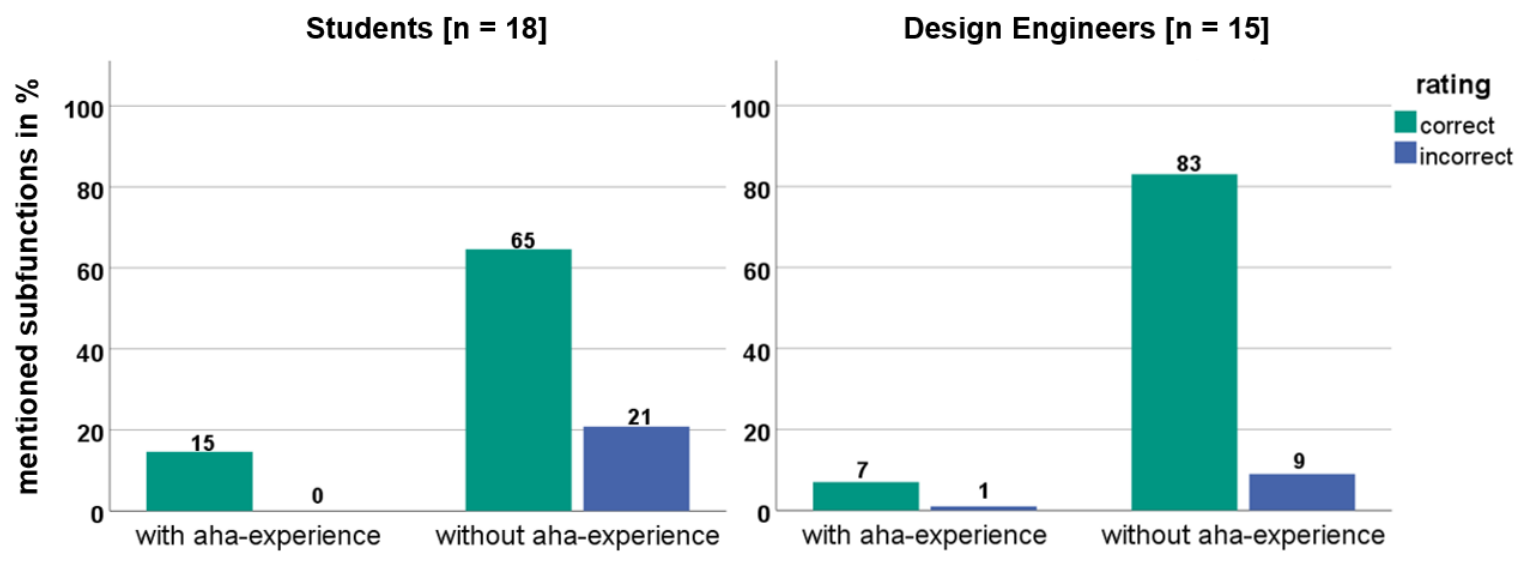

Figure 2. Relation between aha-experience and correctness of the solution for students $[n=18]$ and design engineers $[n=15]$.

\subsection{Mental impasse}

Considering the subfunctions where aha-experiences were observed, it is noticeable that the majority is concentrated on only three functions. Thus, out of 27 aha-experiences, 8 occurred in subfunction 4 "limiting swivel range", followed by subfunction 1 "generate rotation" with 7 situations and subfunction 3 " brace rotation" with 4 situations. This means that $70 \%$ of the aha-experiences occurred among only 3 subfunctions. Table 1 contains representative statement on aha-experiences for the three subfunctions mentioned above. 


\section{Subfunction \\ (participant)}

limiting swivel range (SF 4) (design engineer 7)

generate rotation $(\mathrm{SF} 1)$

(student 18)

brace rotation (SF 3)

(student 13)

\section{Statement of the participant}

Oh exactly, these ["adjustment rings"] were mounted the wrong way. Now the pin hits the respective stop in both directions of rotation. Now it becomes clearer.

When it's open, I guess, water comes through here [front pipe] that.... Aaahhh. Okay, okay. The water then goes here in front [front pipe], comes out here and behind the whole gear is here again a gear wheel with bigger blades [turbine] and, yes, if just, if a pipe is open, then water flows through here and then drives this wheel [turbine] at the end in one direction.

Oh, this whole component [gearbox] rotates as well, doesn't it? Ahh ok. So, this whole part [gearbox]here is probably turning like this [turns gearbox housing in frame]. ahh and then it turns on. Ok.

So the task is done when I have understood how it works? Ok yes, then I am done.

Furthermore, concerning research question 2, it was evaluated how much time the participants needed to identify the next subfunction, measured from the time when the previous subfunction was formulated. Figure 3 shows how much time it took the participants to identify a subfunction on average. Again, the cases with and without aha-experience were distinguished and students and design engineers were considered separately.

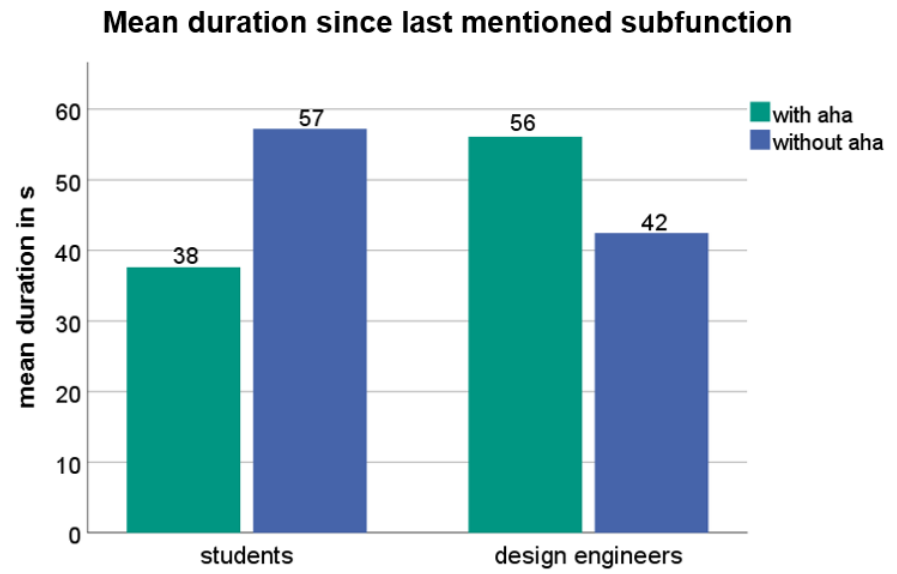

Figure 3. Mean time in seconds between two mentioned subfunctions for students [n = 18] and design engineers [ $n=15]$

While there is no statistically significant difference between the two categories with and without ahaexperience among the students, this can be shown very clearly among the designers. The duration preceding a detected subfunction is significantly higher when it is accompanied by an aha-experience. (two-tailed Mann-Whitney U test, $\mathrm{U}=270.5, \mathrm{p}=0.47$ )

Taking a closer look at the graphs in Figure 4, it is interesting to note that in both groups the peak of subfunctions that are identified without aha-experience takes less time than a function that is identified with aha-experience. However, this difference is much less visible among students than among design engineers. 

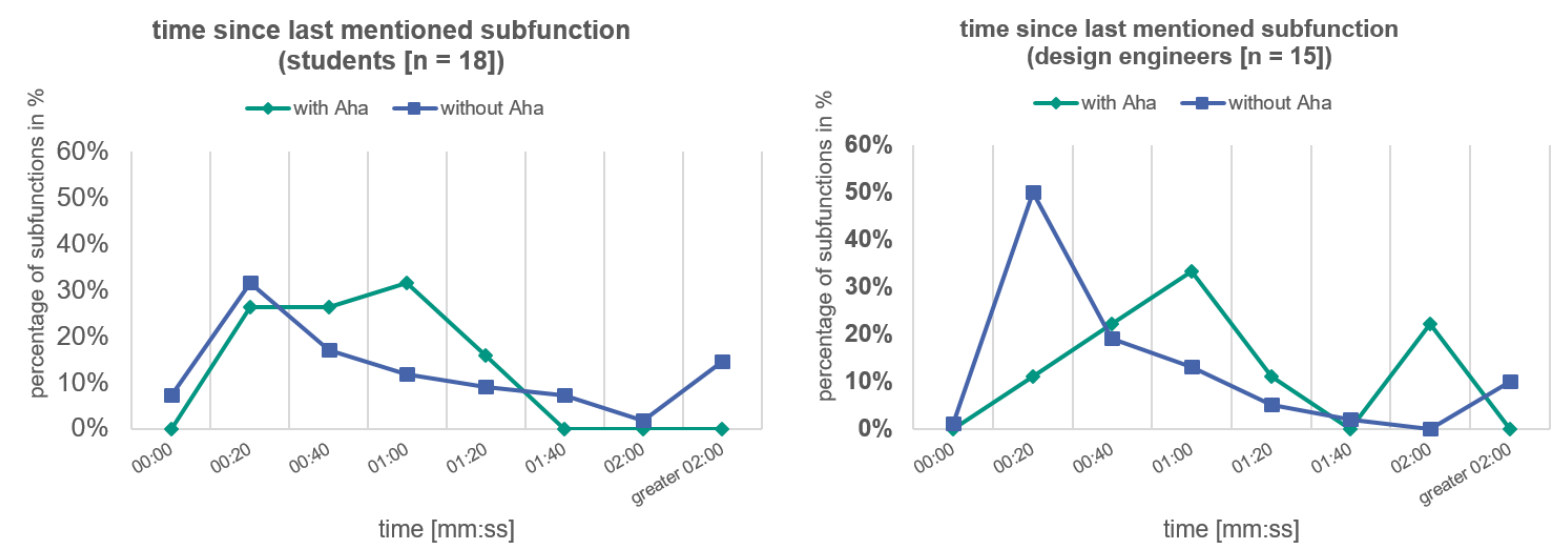

Figure 4. Time between two mentioned subfunctions for students [ $n=18]$ and design engineers $[n=15]$

\section{DISCUSSION}

As described in the state of research, there have not yet been any findings as to whether and to what extent aha-experiences have an impact on correct identified subfunctions in functional analysis in design. The results of this research indicate that aha-experiences do occur in functional analysis. In addition, a positive relation to the correctness of a solution was found within the group of students when the identification of the subfunction was accompanied by an aha-experience (see Figure 2). This result also corresponds with previous findings from the current state of research, according to which solutions involving an aha-experience are more often correct (Danek and Salvi, 2018). Research question 1 can therefore be answered by showing that aha-experiences have a positive effect on the correctness of solutions in functional analysis.

Furthermore, there seem to be some subfunctions that particularly often trigger aha-experiences. Especially the adjustment of the swivel range by the adjustment rings tends to trigger an aha-experience among the participants. However, the reasons for this phenomenon could not be determined from the data. A potential explanatory approach is that this subfunction marks an interface between the gear unit and the housing of the lawn sprinkler. At this location, the separate consideration of the two subsystems could particularly often lead to a mental impasse, which is then overcome by an insight that is accompanied by an aha-experience. This is most likely caused by the combination of the two subsystems which initiates a restructuring process as it is described by Bilalic et al. (2019). Another interesting finding of this investigation is that the design engineers had considerably fewer aha-experiences than the students who did not have such a large amount of experience. A possible explanation for this might be that the task was easier for the design engineers and therefore fewer mental impasses were created that could be overcome by an insight and an aha-experience that accompanied this insight. The engineering designers seem to have had considerably less problems in completing the task, which can be seen in the higher number of quickly identified subfunctions without aha-experience. However, once a mental impasse arose, it took them significantly longer to overcome it. This is also supported by the fact that the design engineers required considerably more time for the few subfunctions that they identified with aha-experiences as can be seen in Figure 4. In contrast, this difference cannot be seen so clearly in the data of the students. The reason for this could be that the error rate among students was significantly higher than among engineering designers. While designers are aware of the fact that a solution found must be correct in all circumstances, students tend to use the first best solution found.

Further investigation of the aha-experience in the context of the design is needed to investigate the triggers of the phenomenon of the aha-experience in more detail. In the future, further techniques for detecting aha-experiences could be used to increase the objectivity of the evaluation. For example, it would be possible to use questionnaires on which the participants themselves could report ahaexperiences. Another approach would be the use of physiological signals. Especially there are already promising approaches using eye-tracking as a measuring method (Salvi et al., 2020).

In addition, further research should be conducted on possible triggers of aha-experiences during functional analysis. Since the data presented here already suggest that solutions are more often correct when accompanied by an aha-experience, the triggers of these aha-experiences could be used to 
develop design methods for design engineers and engineering design students. Such methods do not necessarily have to be developed from the beginning. If the triggers of aha-experiences are known, existing methods, which have already been proven to be beneficial, can be further developed by adding new elements. For example, methods for the identification of embodiment function relations can be adapted in such a way that aha-experiences occur more frequently.

\section{REFERENCES}

Akin, O. and Akin, C. (1996), "Frames of reference in architectural design: analysing the hyperacclamation (Aha-!)", Design Studies, Vol. 17 No. 4, pp. 341-361.

Ash, I.K., Jee, B.D. and Wiley, J. (2012), "Investigating Insight as Sudden Learning", The Journal of Problem Solving, Vol. 4 No. 2. 10.7771/1932-6246.1123.

Badke-Schaub, P. and Frankenberger, E. (1999), “Analysis of design projects”, Design Studies, Vol. 20 No. 5 , pp. 465-480. 10.1016/S0142-694X(99)00017-4.

Bilalić, M., Graf, M., Vaci, N. and Danek, A.H. (2019), "The temporal dynamics of insight problem solving restructuring might not always be sudden”, Thinking \& Reasoning, Vol. 11 No. 3, pp. 1-37. 10.1080/13546783.2019.1705912.

Bock, A. (1955), "Die Begriffe "Konstruieren, Entwerfen und Gestalten"”, Die Technik: technischwissenschaftliche Zeitschrift für Grundsatz- und Querschnittsfragen, Vol. 10 No. 8, pp. 504-505.

Booth, J.W., Reid, T.N., Eckert, C. and Ramani, K. (2015), "Comparing Functional Analysis Methods for Product Dissection Tasks”, Journal of Mechanical Design, Vol. 137 No. 8, p. 27. 10.1115/1.4030232.

Bühler, K. (1907), “Tatsachen zu einer Psychologie der Denkvorgänge. I Über Gedanken”, Archiv für die gesamte Psychologie, Vol. 9, pp. 297-365.

Chrysikou, E.G. and Gero, J.S. (2020), "Using neuroscience techniques to understand and improve design cognition”, AIMS Neuroscience, Vol. 7 No. 3, pp. 319-326. 10.3934/Neuroscience.2020018.

Cohen, J. (1960), “A Coefficient of Agreement for Nominal Scales”, Educational and sychological measurement, Vol. 20 No. 1, pp. 37-46.

Cranford, E.A. and Moss, J. (2012), "Is Insight Always the Same? A Protocol Analysis of Insight in Compound Remote Associate Problems", The Journal of Problem Solving, Vol. 4 No. 2. 10.7771/1932-6246.1129.

Cross, N., "Design cognition: Results from protocol and other empirical studies of design activity", Design knowing and learning: Cognition in design education, pp. 79-103.

Danek, A.H., Fraps, T., Müller, A. von, Grothe, B. and Ollinger, M. (2013), “Aha! experiences leave a mark: facilitated recall of insight solutions”, Psychological Research, Vol. 77 No. 5, pp. 659-669. 10.1007/s00426-012-0454-8.

Danek, A.H., Fraps, T., Müller, A. von, Grothe, B. and Ollinger, M. (2014), "Working wonders? investigating insight with magic tricks", Cognition, Vol. 130 No. 2, pp. 174-185. 10.1016/j.cognition.2013.11.003.

Danek, A.H. and Salvi, C. (2018), "Moment of Truth: Why Aha! Experiences are Correct", The Journal of Creative Behavior, Vol. 9, pp. 322-324. 10.1002/jocb.380.

Danek, A.H. and Wiley, J. (2016), "What about False Insights? Deconstructing the Aha! Experience along Its Multiple Dimensions for Correct and Incorrect Solutions Separately”, Frontiers in Psychology, Vol. 7, p. 2077. 10.3389/fpsyg.2016.02077.

Eckert, C., Ruckpaul, A., Alink, T. and Albers, A. (2012), "Variations in functional decomposition for an existing product: Experimental results", Artificial Intelligence for Engineering Design, Analysis and Manufacturing, Vol. 26 No. 2, pp. 107-128. 10.1017/S0890060412000029.

Ericsson, K.A. and Simon, H.A. (1993), Protocol analysis - Verbal reports as data, MIT Press, Cambridge, Massachusetts.

Fleck, J.I. and Weisberg, R.W. (2004), "The use of verbal protocols as data: An analysis of insight in the candle problem", Memory \& Cognition, Vol. 32 No. 6, pp. 990-1006.

Gericke, K. and Blessing, L. (2012), "An analysis of design process models across disciplines", DS 70: Proceedings of DESIGN 2012, the 12th International Design Conference, Dubrovnik, Croatia.

Hacker, W. (2002), “Konstruktives Entwickeln: Psychologische Grundlagen”, in Hacker, W. (Ed.), Denken in der Produktentwicklung: psychologische Unterstützung der frühen Phasen, vdf Hochschulverlag AG \& Rainer Hampp, Zurich, Stuttgart, pp. 11-25.

Hess, S., Lohmeyer, Q. and Meboldt, M. (2018), "Mobile Eye Tracking in Engineering Design Education”, Design and Technology Education: an International Journal, Vol. 23 No. 2, pp. 86-98.

Jones, G. (2003), "Testing Two Cognitive Theories of Insight”, Journal of Experimental Psychology: Learning, memory and cognition, Vol. 29 No. 5, p. 1017.

Landis, J.R. and Koch, G.G. (1977), "The measurement of observer agreement for categorical data", Biometrics, pp. 159-174.

Laukkonen, R.E., Kaveladze, B.T., Tangen, J.M. and Schooler, J.W. (2020), "The dark side of Eureka: Artificially induced Aha moments make facts feel true", Cognition, Vol. 196, pp. 104-122. 10.1016/j.cognition.2019.104122. 
Matthiesen, S. (2011), "Seven years of product development in Industry-experiences and requirements for supporting engineering design with "Thinking Tools"”, Proceedings of the 18th International Conference on Engineering Design (ICED 11), Impacting Society through Engineering Design, Vol. 9: Design Methods and Tools pt. 1, Lyngby/Copenhagen, Denmark, 15.-19.08. 2011.

Matthiesen, S. and Nelius, T. (2018a), "Eye tracking study on successful micro-strategies by design engineers for the synthesis-driven analysis of technical systems", in Horváth, I., Suárez Rivero, J.P. and Hernández Castellano, P.M. (Eds.), Proceedings of TMCE 2018, 7-11 May, 2018, Las Palmas de Gran Canaria, Gran Canaria, Spain, TU Delft, Delft, 295-303.

Matthiesen, S. and Nelius, T. (2018b), "Managing Assumptions during Analysis - Study on successful Approaches of Design Engineers", in Ekströmer, P., Schütte, S. and Ölvander, J. (Eds.), DS 91: Proceedings of NordDesign 2018, Linköping, Sweden, 14th - 17th August 2018, The Design Society, Glasgow.

Matthiesen, S., Nelius, T., Pflegler, B. and Gutmann, T. (2017), "Studiendesign zur Untersuchung der synthesegetriebenen Analyse von Konstrukteuren", DFX-Symposium, Bamberg.

McHugh, M.L. (2012), "Interrater reliability: the kappa statistic", Biochemia medica: Biochemia medica, Vol. 22 No. 3, pp. 276-282.

Meboldt, M., Matthiesen, S. and Lohmeyer, Q. (2013), “The Dilemma of Managing Iterations in Time-to-market Development Processes", Second International Workshop on the Modelling and Management of Engineering Processes (MMEP 2012). 10.3929/ETHZ-A-009774163.

Nelius, T., Doellken, M., Zimmerer, C. and Matthiesen, S. (2020), "The impact of confirmation bias on reasoning and visual attention during analysis in engineering design: An eye tracking study”, Design Studies, Vol. 71. 10.1016/j.destud.2020.100963.

Ruckpaul, A., Nelius, T. and Matthiesen, S. (2015), "Differences in analysis and interpretation of technical systems by expert and novice engineering designers", Proceedings of the 20th International Conference on Engineering Design, Vol. 20, pp. 339-348.

Salvi, C., Bricolo, E., Kounios, J., Bowden, E. and Beeman, M. (2016), "Insight solutions are correct more often than analytic solutions", Thinking \& Reasoning, Vol. 22 No. 4, pp. 443-460. 10.1080/13546783.2016.1141798.

Salvi, C., Simoncini, C., Grafman, J. and Beeman, M. (2020), "Oculometric signature of switch into awareness? Pupil size predicts sudden insight whereas microsaccades problem-solving via analysis”, NeuroImage, pp. 116-933. 10.1016/j.neuroimage.2020.116933.

Wynn, D.C. and Eckert, C.M. (2017), "Perspectives on iteration in design and development", Research in Engineering Design, Vol. 28 No. 2, pp. 153-184. 10.1007/s00163-016-0226-3. 